\title{
Serosurvey for Health-Care Workers Provides Supportive Evidence for the Effectiveness of Hydroxychloroquine Prophylaxis against SARS-CoV-2 Infection
}

\author{
Reetika Malik Yadavi,(D), Archana Pate ${ }^{3}$, Aruna Shankarkumar',(D), Shreyasi Athalye ${ }^{2,(\mathbb{D},}$ \\ Shweta Shinde ${ }^{1}$, Umair Ahmed Bargir ${ }^{1, \mathbb{D}}$, Mangesh Pate ${ }^{3}$, Makarand Ganpule ${ }^{3}$, Meena Pruthi ${ }^{3}$, \\ Hemant Patil ${ }^{3}$, Manisha Rajan Madkaikar ${ }^{1, *}$ (D) \\ ${ }^{1}$ Department of Pediatric Immunology and Leukocyte Biology, ICMR-National Institute of Immunohematology, 13th floor, \\ New Multi-storeyed Building, KEM Hospital Complex, Parel, Mumbai 400012, India \\ ${ }^{2}$ Department of Transfusion Transmitted Diseases, ICMR-National Institute of Immunohematology, 13th floor, \\ New Multi-storeyed Building, KEM Hospital Complex, Parel, Mumbai 400012, India \\ ${ }^{3}$ Indian Medical Association Dombivli, IMA Hall, 2nd floor, Deepshikha CHS, Dombivli 421201, India
}

\section{ARTICLE INFO}

Article History

Received 08 October 2020

Accepted 15 April 2021

\section{Keywords}

Sero-surveillance

SARS-CoV-2

healthcare workers

hydroxychloroquine

prophylaxis

\begin{abstract}
Background: The Severe Acute Respiratory Syndrome Coronavirus 2 (SARS-CoV-2) pandemic has resulted in occupational exposure among Healthcare Workers (HCWs) and a high risk of nosocomial transmission. Asymptomatic infection and transmission of infection before the development of symptoms are well-recognized factors contributing to the spread of infection. We conducted a cross-sectional observational study to understand the seroprevalence of SARS-CoV-2 infection among HCWs and to verify the appropriateness of infection control measures, particularly Hydroxychloroquine (HCQ) prophylaxis.

Methods: A cross-sectional sero-surveillance study was conducted among $500 \mathrm{HCWs}$ in Dombivli and surrounding Mumbai Metropolitan area (Maharashtra, India) between 21st July and 3rd August 2020. The vulnerability of the study participants to SARS-CoV-2 infection was ascertained through a history of (i) involvement in direct care, (ii) exposure to aerosol-generating procedures, (iii) co-morbidities, (iv) Personal Protective Equipment (PPE) use, and (v) HCQ prophylaxis. SARS-CoV-2 IgG antibodies were tested using COVID KAVACH anti-SARS-CoV-2 IgG antibody detection enzyme-linked immunosorbent assay (ELISA) from Zydus Cadila. A systematic analysis of the correlation between the development of antibodies and factors affecting vulnerability to infection was performed.
\end{abstract}

Results: The overall SARS-CoV-2 seroprevalence in the study population was $11 \%$. Providing direct care to COVID-19 patients (Adjusted OR 16.4, 95\% CI 3.3-126.9, $p=0.002$ ) for long hours and irregular use of PPE (Adjusted OR 3.78, 95\% CI 1.1-11.9, $p=0.02$ ) were associated with an increased incidence of seropositivity. Prophylaxis with HCQ may have a role in reducing the vulnerability to infection as depicted by univariate and multivariate analysis (Adjusted OR 0.55, 95\% CI 0.3-0.9, $p=0.047$ ). It was also noted that those not on HCQ prophylaxis were threefold more prone to infection and developed severe disease as compared to those on HCQ prophylaxis.

Conclusion: Prophylaxis with HCQ may have a role in mitigating the incidence and severity of SARS-CoV-2 infection. Although vaccination is the most robust strategy to safeguard against COVID-19, it will be months before vaccination percolates to the masses. In the face of the second wave of COVID-19, the use of HCQ prophylaxis in combination with use of face-masks regularly may be considered as a cost-effective measure for population dense areas like urban slums where social distancing is not possible.

(C) 2021 The Authors. Published by Atlantis Press International B.V

This is an open access article distributed under the CC BY-NC 4.0 license (http://creativecommons.org/licenses/by-nc/4.0/)

\section{INTRODUCTION}

The Severe Acute Respiratory Syndrome Coronavirus 2 (SARS$\mathrm{CoV}-2$ ) pandemic has resulted in occupational exposure among Healthcare Workers (HCWs) and a high risk of nosocomial transmission [1]. The WHO Weekly Epidemiological Update (2nd February 2021), reported over 1.29 million infections with SARSCoV-2 among HCWs across 183 countries by 31st January 2021 accounting for approximately $8 \%$ of cases [2]. Early reports from

"Corresponding author.Email: madkaikarmanisha@gmail.com

Data availability statement: The authors confirm that the data supporting the findings of this study are available within the article [and/or] its supplementary materials.
China and Italy had mentioned that $29 \%$ and $9 \%$ respectively of the total cases were HCWs [3,4]. SARS-COV-2 testing for symptomatic HCWs in UK, Netherland, and India revealed SARS-CoV-2 infection in $18 \%, 11 \%$ and $5 \%$ of the tested HCWs respectively [5-7].

Healthcare workers had not been well prepared for the outbreak especially in departments other than infectious diseases, and the general lack of awareness among the staff to take precautions and inadequate training on the use of Personal Protective Equipment (PPE) added to the situation [8]. Also, asymptomatic infection and transmission of infection before the development of symptoms are well-recognized factors contributing to the spread of infection [9]. Laboratory testing based on reverse transcription polymerase 
chain reaction (RT-PCR) does not necessarily reflect on the infectivity among HCWs [10]. The availability of serological tests for anti-SARS-CoV-2 antibody determination has made it possible to study the seroprevalence and seroconversion in this high-risk population [11]. Some studies on seroprevalence among HCWs in Wuhan, Germany, and Italy have reported it to be $3.8 \%, 1.6 \%$ and $3.4 \%$ respectively [12-14]. The importance of understanding the dynamics of transmission in HCWs lies in planning strategies for the reduction of nosocomial spread.

The search for a medication that would reduce the risk of infection has led to the repurposing of existing medications [15]. One such drug is Hydroxychloroquine (HCQ) which has attracted the attention of the scientific community globally, and Randomized Controlled Trials (RCTs) are underway to assess the efficacy of HCQ in providing effective prophylaxis to those at high risk of contracting the disease [16]. HCQ prophylaxis is recommended by the Indian Council of Medical Research (ICMR) National Taskforce for COVID-19 for asymptomatic HCWs [17].

Some studies on postexposure therapy with HCQ conclude that it did not prevent SARS-CoV-2 infection or symptomatic COVID-19 in healthy persons exposed to a PCR-positive case patient $[18,19]$. The question of preventing COVID-19 with HCQ prophylaxis remained unanswered by initial results of RCT conducted by GrauPujol et al. [20] and Rajasingham et al. [21] as the studies were underpowered.

We report a cross-sectional sero-surveillance study conducted among HCWs in Dombivli and the surrounding area which is a part of the Mumbai Metropolitan Area (MMR) in India. Our study aimed to understand the seroprevalence of SARS-CoV-2 infection among HCWs and to verify the appropriateness of infection control measures, particularly pre-exposure prophylaxis with HCQ.

\section{MATERIALS AND METHODS}

\subsection{Design}

A cross-sectional sero-surveillance study was conducted among HCWs in Dombivli and the surrounding areas (MMR, Maharashtra, India) between 21st July and 3rd August 2020 as a collaborative study by the Indian Medical Association Dombivli and ICMRNational Institute of Immunohaematology and the data was analysed in August 2020.

\subsection{Participant Details and Study Procedure}

Healthcare workers involved in the care of suspected and confirmed COVID-19 patients at dedicated COVID hospitals in Dombivli area were invited to participate in the study. They were requested to fill a survey questionnaire adopted from the WHO risk assessment tool for exposed HCWs (WHO/2019-nCoV/ HCW_risk_assessment/2020.2) after informed consent. The vulnerability of the study participants to SARS-CoV-2 infection was ascertained through a history of (i) place of duty and involvement in the direct care of COVID-19 patients, (ii) performing or exposure to aerosol-generating procedures, (iii) presence of co-morbidities, (iv) use of PPE, and (v) use of immune-modulators which was collected through a structured questionnaire. A history of the development of symptoms of a viral illness in the preceding 3 months and RT-PCR testing were also recorded. Only those who completed the survey questionnaire were included in the study. About $3 \mathrm{ml}$ blood was collected per participant and tested for SARS CoV-2 IgG antibodies using COVID KAVACH anti-SARS-CoV-2 IgG antibody detection ELISA from Zydus Cadila.

\subsection{HCQ Prophylaxis}

Hydroxychloroquine prophylaxis was defined as an intake of HCQ by asymptomatic HCWs as recommended by the ICMR National Taskforce for COVID-19, with a loading dose of $400 \mathrm{mg}$ twice a day on day 1 followed by $400 \mathrm{mg}$ weekly. The HCWs who consumed HCQ were divided into four groups - No HCQ, and intake of HCQ for $<6$ weeks, $6-10$ weeks and $>10$ weeks. Participants who did not indicate the number of weeks of intake of HCQ prophylaxis were not considered for purpose of regression analysis.

\subsection{Ethical Approval}

The study is approved by the Institutional Ethics Committee of ICMR-National Institute of Immunohaematology.

\subsection{Analysis}

The data were collated using Microsoft Excel, and statistical analysis was done using Graph pad prism8 (Chicago, IL, USA) for Microsoft Windows. Primary univariate analysis was performed using the Chi-square and Fisher's exact tests to correlate the development of antibodies and the factors affecting vulnerability. A secondary analysis was performed using multivariate logical regression for factors that significantly affected vulnerability to infection according to the primary analysis.

\section{RESULTS}

Of the 560 HCWs who were provided with the survey questionnaire, 500 who completed the survey were included in the study. The characteristics of the participant group and history of a viral illness are summarised in Table 1.

Overall, 11\% (55/500) participants were detected with IgG antibodies to SARS-CoV-2. About two-fifth (33/55) of those with IgG antibodies had experienced symptoms of a viral infection in the preceding 3 months. Among the symptomatic participants, 23/33 underwent RT-PCR testing, 14 (60.9\%) were confirmed with COVID-19 disease and treated as per standard treatment guidelines.

A comparison of the seroprevalence between HCWs positioned in dedicated COVID-19 care and those working in non-COVID-19 areas revealed a higher seroprevalence of $17.2 \%$ among those working in non-COVID-19 areas as compared to $9.6 \%$ among those providing direct care to COVID-19 patients. In univariate analysis, the variables which affected vulnerability to infection, in a statistically significant manner include the number of hours of exposure, rational use of PPE, and HCQ prophylaxis as depicted in Table 2. The development of IgG antibodies correlated well with the number of hours of exposure $(p=0.002)$ as shown in Figure 1. 
Table 1 Participant characteristics and history of symptoms

\begin{tabular}{|c|c|c|c|c|}
\hline S. No. & Characteristics & $\begin{array}{l}\text { Sero-positive } \\
\quad(n=55)\end{array}$ & $\begin{array}{l}\text { Sero-negative } \\
\quad(n=445)\end{array}$ & $p$ \\
\hline 1 & Median age (in years) & 40 & 44 & \\
\hline \multirow[t]{3}{*}{2} & Sex & & & \\
\hline & Male & $67.3 \%$ & $58.4 \%$ & 0.2 \\
\hline & Female & $32.7 \%$ & $41.6 \%$ & \\
\hline \multirow[t]{6}{*}{3} & Primary place of work & & & \\
\hline & $\begin{array}{l}\text { Out-Patient } \\
\text { department/ } \\
\text { Pharmacy/X-ray } \\
\text { department/ECG }\end{array}$ & $12.4 \%$ & $87.6 \%$ & 0.9 \\
\hline & $\begin{array}{l}\text { Medical unit/ICU/ } \\
\text { Emergency/OT/ } \\
\text { Either or all }\end{array}$ & $10.2 \%$ & $89.8 \%$ & \\
\hline & Laboratory & $13.5 \%$ & $86.5 \%$ & \\
\hline & Administration & $8.7 \%$ & $91.3 \%$ & \\
\hline & $\begin{array}{l}\text { Others including } \\
\text { teleconsultation and } \\
\text { support services } \\
\text { like an attendant, } \\
\text { ambulance services, } \\
\text { security, etc. }\end{array}$ & $9.8 \%$ & $90.1 \%$ & \\
\hline \multirow[t]{5}{*}{4} & Co-morbidities & & & \\
\hline & Diabetes & $10.9 \%$ & $14.3 \%$ & 0.6 \\
\hline & $\begin{array}{l}\text { Hypertension/Heart } \\
\text { disease }\end{array}$ & $16.3 \%$ & $16.6 \%$ & \\
\hline & Asthma/Lung disease & $3.6 \%$ & $1.6 \%$ & \\
\hline & Others & $5.4 \%$ & $6.7 \%$ & \\
\hline \multirow[t]{2}{*}{5} & $\begin{array}{l}\text { Type of exposure to } \\
\text { COVID-19 patients }\end{array}$ & & & \\
\hline & Direct & $81.8 \%$ & $78.4 \%$ & 0.8 \\
\hline 6 & $\begin{array}{l}\text { Presence of symptoms } \\
\text { consistent with a viral } \\
\text { illness in the preceding } \\
\text { three months }\end{array}$ & 33 & 152 & 0.0003 \\
\hline 7 & Tested with RT-PCR & $69.7 \%$ & $41.4 \%$ & NA \\
\hline \multirow[t]{3}{*}{8} & SARS-CoV-2 RT-PCR & & & \\
\hline & Test positive & $60.9 \%$ & $17.5 \%$ & \\
\hline & Test negative & $39.1 \%$ & $82 \cdot 5 \%$ & \\
\hline 9 & $\begin{array}{l}\text { HCWs reporting } \\
\text { affected family } \\
\text { members }(n=200)\end{array}$ & $2.5 \%$ & $3 \%$ & NA \\
\hline
\end{tabular}

Running Table 2 HCQ prophylaxis numbers through logistic regression, for each increasing category of HCQ use we noted a dose-response OR $=0.70$ (95\% CI 0.50-0.99, $p=0.032)$. This trend is much stronger among the ever-exposed categories (i.e., excluding the never-used HCQ people), per-category OR $=0.27$ (95\% CI $0.11-0.65, p=0.0036)$. None of the participants who took HCQ prophylaxis reported serious side effects (specifically arrhythmias).

Multivariate analysis for factors that might affect the development of antibodies, namely direct contact with COVID-19 patients, use of PPE, and HCQ prophylaxis was performed using multivariate logical regression. Those who had provided direct care to COVID-19 patients had sixteen times the odds of developing antibodies (95\% CI 3.3-126.9, $p=0.002$ ) than those working in nonCOVID areas, and non-usage of PPE increased the odds of infection by almost four times (95\% CI 1.1-11.9, $p=0.02$ ). HCQ prophylaxis was associated with reduced odds of developing antibodies to almost half (95\% CI 0.3-0.9, $p=0.047)$ as depicted in Table 3.
Table 2 Analysis of factors affecting vulnerability and statistical significance for their differences $(p)$ in univariate analysis

\begin{tabular}{lccc}
\hline Variables & $\begin{array}{c}\text { SARS-CoV-2 } \\
\text { antibody } \\
\text { positive }(\boldsymbol{n}=\mathbf{5 5})\end{array}$ & $\begin{array}{c}\text { SARS-CoV-2 } \\
\text { antibody }\end{array}$ & $\boldsymbol{p}$ \\
\hline negative $(\boldsymbol{n}=\mathbf{4 4 5})$ & \\
\hline Rational use of & $85.4 \%$ & $71.5 \%$ & $\mathbf{0 . 0 4}^{*}$ \\
$\quad$ PPE as per WHO & & & \\
$\quad$ recommendations & & & \\
No. of hours of exposure & $24.4 \%$ & $19.5 \%$ & $\mathbf{0 . 0 0 2}^{* *}$ \\
$>8$ & $44.4 \%$ & $22.3 \%$ & \\
$4-8$ & $31.1 \%$ & $58.2 \%$ & \\
$<4$ & $22.7 \%$ & $18.8 \%$ & 0.55 \\
Exposure to aerosol- & & & \\
generating procedures & & & \\
HCQ prophylaxis & & $11.8 \%$ & $\mathbf{0 . 0 1}^{*}$ \\
HCQ $<6$ weeks & $25 \%$ & $22.8 \%$ & \\
6-10 weeks & $11.4 \%$ & $10.7 \%$ & \\
$>10$ weeks & $2.3 \%$ & $54.5 \%$ & \\
No HCQ & $61.4 \%$ & 97 & $>0.99$ \\
\hline Presence of co-morbidity & 12 & & \\
\hline
\end{tabular}

${ }^{a}$ Only those providing direct care were included in analysis $(n=45$ for the sero-positive group and $n=349$ for the sero-negative group). ${ }^{\mathrm{b}}$ Data regarding the number of weeks of intake of HCQ was missing for $101 \mathrm{HCW}$, hence excluded from the analysis. Boldface $p$-value indicates statistical significance $(" p<0.05, " * 0<0.01)$.

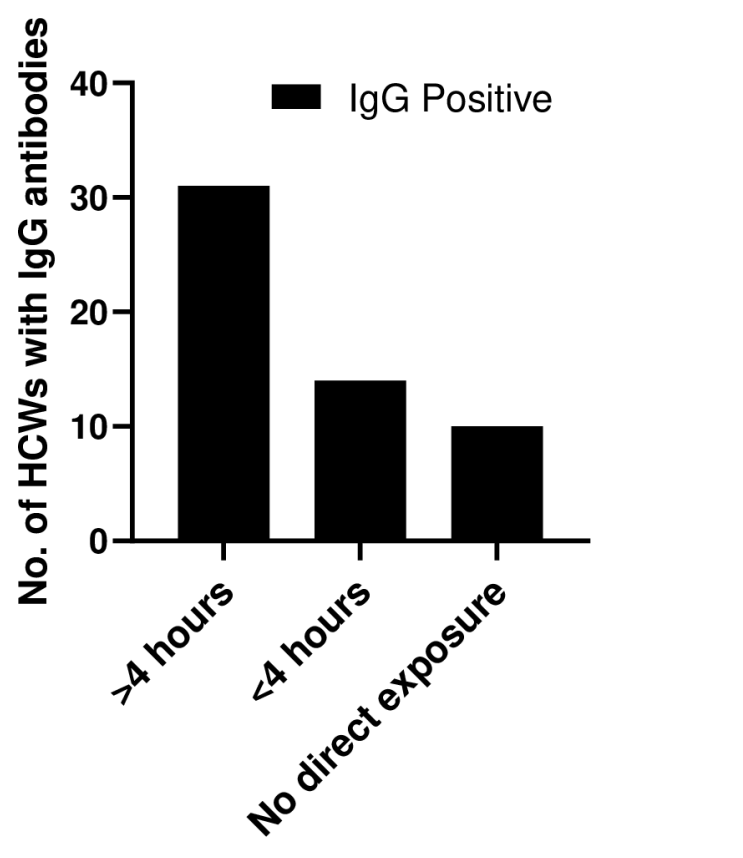

No. of hours of exposure

Figure 1 Correlation of the number of hours of exposure with the development of IgG antibodies.

Amongst participants who were on HCQ prophylaxis $(n=279)$, 111 reported symptoms suggestive of a viral illness in the preceding 3 months, SARS-CoV-2 infection was confirmed by RT-PCR testing in seven $(2.5 \%)$, and $2 / 7$ required hospitalization. However, both of them had mild symptoms, did not require supplemental oxygen, and were hospitalized only for observation. On the other hand, 78 among those not on HCQ prophylaxis $(n=221)$ were symptomatic, SARS-CoV-2 infection was confirmed in 13 (5.9\%) by RT-PCR, and 9/13 required hospitalization. All that hospitalized 
Table 3 Odds ratios of the presence of anti-SARS-CoV-2 antibody for study variables in multivariate logistic regression analysis Dependent variable: Positive SARS-CoV-2 antibody test Analysis of independent variables:

\begin{tabular}{lclc}
\hline $\begin{array}{l}\text { Factor affecting } \\
\text { vulnerability to } \\
\text { infection }\end{array}$ & Adjusted odds ratio & $\mathbf{9 5 \%}$ CI & $\boldsymbol{p}$ \\
\hline Direct care & 16.40 & $\mathbf{3 . 3 - 1 2 6 . 9}$ & $\mathbf{0 . 0 0 2}$ \\
Never used PPE & 3.78 & $1.1-11.9$ & $\mathbf{0 . 0 2 0}^{*}$ \\
HCQ prophylaxis & 0.55 & $\mathbf{0 . 3 - 0 . 9}$ & $\mathbf{0 . 0 4 7}$ \\
\hline
\end{tabular}

Boldface $p$-value indicates statistical significance $\left({ }^{*} p<0.05,{ }^{* *} p<0.01\right)$.

required low molecular weight heparin and steroids in addition to the standard treatment, and supplemental oxygen was required by two among those admitted.

A total of $71(14.2 \%)$ participants reported a prior SARS-CoV-2 RT-PCR test. All participants who had a prior nucleic acid SARSCoV-2 test were either symptomatic at the time of testing or among contacts of confirmed cases, which is consistent with the local practice. Of the 71 participants, 25 tested positive; however, only $14 / 25$ participants were seropositive for SARS-CoV-2 IgG antibodies, and $11 / 25$ were seronegative.

Of 200 participants who provided information on affected family members, 11 a reported an affected family member.

\section{DISCUSSION}

The overall seroprevalence of SARS-CoV-2 in the study population was $11 \%$. Only about two-fifths of the HCWs who had detectable anti-SARS-CoV-2 antibodies reported symptoms consistent with a prior viral illness. This reiterates that testing only symptomatic may miss a substantial number of infected HCWs. This may have implications on nosocomial transmission. Also, 99/189 HCWs from both seropositive and seronegative groups who had experienced symptoms of a viral illness did not undergo RT-PCR testing due to the presence of mild or non-specific symptoms. However, $12(12.1 \%)$ of these had detectable antibodies. As such, HCWs being a high-risk group should not ignore even mild symptoms and should undergo testing.

Comparing the results with sero-prevalence studies from other countries, our study reveals a higher seroprevalence compared to studies from Wuhan, Germany, and Italy [12-14]. This might be indicative of complacence in observing strict precautions at all times, especially in non-COVID-19 wards and non-testing despite the presence of symptoms.

Four of the $11 \mathrm{HCW}$ s who were seronegative despite SARS-CoV-2 infection confirmed by RT-PCR testing had less than 3 weeks' duration between symptom onset and testing which might be responsible for a negative result. Three participants were asymptomatic and detected RT-PCR positive on contact screening, and one participant had reported mild symptoms, as such they might have titres below detectable levels. This has been observed in a recent study which reported low neutralizing antibody titre in as many as 30\% individuals recovering from mild SARS-CoV-2 infection. Three participants had borderline OD values; and a repeat testing might be required in these participants.
Direct care to COVID-19 patients for long hours was associated with an increased incidence of seropositivity; this might be attributed to overcrowding in the OPD and emergency areas, exposure to aerosol-generating procedures, and suboptimal use of PPE. Optimizing working conditions by adjusting shift schedules, following strict precautionary measures like hand hygiene practices, and regular use of PPE may help reduce the risk of infection [22]. Strict adherence to strategies for RT-PCR testing in case of symptoms and quarantine of confirmed SARS-CoV-2 infected HCWs may also help in limiting the risk of nosocomial transmission.

Prophylaxis with HCQ may have a role in reducing the vulnerability to infection $[7,23]$ as depicted by univariate and multivariate analysis. It was also noted that those not on HCQ prophylaxis were threefold more prone to infection as confirmed by RT-PCR as compared to those on HCQ prophylaxis. Thus, HCQ may even have a role in mitigating the severity of the disease among those infected.

Hydroxychloroquine inhibits endosomal acidification thereby elevating the $\mathrm{pH}$ of endosomes and prevents replication of SARSCoV-2. Also, the drug appears to interfere with terminal glycosylation of the cellular receptor, Angiotensin converting enzyme (ACE-2) thereby affecting the virus-receptor binding and entry of virus into the cell. The immunomodulatory effect may also help in preventing cytokine storm associated with COVID-19 disease [24]. The universal availability, low cost, long half-life and weekly dosing schedule are other benefits that make HCQ an appropriate choice as a prophylactic agent. Although several trials are underway to assess the efficacy of HCQ prophylaxis for SARS-CoV-2 infection, conclusive evidence to support or refute the use of this drug is awaited [16,23]. Our observational study supports the evidence in favour of HCQ prophylaxis which is also reported by three other Indian studies by Chatterjee et al. [7], Goenka et al. [25] and Khurana et al. [26]. Our manuscript shows that there is both a benefit of HCQ ever-use and a dose-response in weeks of use which has also been reported by the other Indian studies mentioned above. A recent review of HCQ PrEP by Stickler and Fesler [27] mentions the decreased rate of COVID-19 mortality in patients with autoimmune diseases taking HCQ. All these observational studies including ours enrolled a total of 2660 patients and provide corroborative evidence of the effectiveness of HCQ prophylaxis for frontline HCWs. None of these studies were RCTs which might be considered a limitation of the study. However, RCTs require a longer time for completion and an average cost of millions of dollars thereby taking a long time to generate any conclusive results.

The effectiveness of the regular use of PPE and HCQ prophylaxis can also be emphasized by comparing seroprevalence among HCWs and the city-wide seroprevalence which was reported in early July to be about 33\% in the city of Mumbai [28]. The results with PPE usage and HCQ pre-exposure prophylaxis can be considered useful in face the second wave of COVID-19. Although vaccination is the most robust strategy to safeguard against COVID-19, it will be months before vaccination percolates to the masses. The use of HCQ prophylaxis in combination with use of face-masks regularly may be considered as a cost-effective measure for population dense areas like urban slums where social distancing is not possible. 


\section{CONFLICTS OF INTEREST}

The authors declare they have no conflicts of interest.

\section{AUTHORS' CONTRIBUTION}

AP contributed in conceptualization, investigation. RMY contributed in methodology, project administration, investigation, data curation and analysis, visualization, writing and editing the manuscript. AS and SA contributed in investigation, validation. SS, Mangesh P, HP, Meena P, and MG contributed in investigation. UAB contributed in designing the data collection tool, resources. MRM contributed in supervision, funding acquisition, resources, validation, review of manuscript and approval of final version for submission.

\section{FUNDING}

The work was supported by the Indian Council of Medical Research (ICMR), an autonomous Government-funded medical research council. The sponsor had no role in study design; collection, analysis, and interpretation of data; writing the report; and the decision to submit the report for publication. The corresponding author who is Director of an ICMR institute was responsible for the study design, supervision of the study, review and approval of the final version of the manuscript. The corresponding author had access to all the data and had final responsibility for the decision to submit for publication.

\section{ACKNOWLEDGMENTS}

1. Dr Vijay Suryavanshi, Commissioner Kalyan Dombivli Municipal Corporation for Administrative approval to conduct the study in Dombivli area.

2. Dr Suresh Kadam, MOH, KDMC for facilitating the study.

3. Technical Officer Mrs. Vaishali Pujari, Technical assistants Mr. Ganeshmoorthy, Mrs. Shreya Chavan and Mr. Ramesh Kawle for support in antibody testing and data entry in excel and Mr. Shailesh Shinde for assistance in sample collection.

\section{REFERENCES}

[1] Koh D. Occupational risks for COVID-19 infection. Occup Med (Lond) 2020;70;3-5.

[2] World Health Organization. Weekly Epidemiological Update. 2021. Available from: https://www.who.int/publications/m/item/ weekly-epidemiological-update---2-february-2021.

[3] Wang D, Hu B, Hu C, Zhu F, Liu X, Zhang J, et al. Clinical characteristics of 138 hospitalized patients with 2019 novel coronavirusinfected pneumonia in Wuhan, China. JAMA 2020;323;1061-9.

[4] Livingston E, Bucher K. Coronavirus disease 2019 (COVID-19) in Italy. JAMA 2020;323;1335.

[5] Keeley AJ, Evans C, Colton H, Ankcorn M, Cope A, State A, et al. Roll-out of SARS-CoV-2 testing for healthcare workers at a large NHS Foundation Trust in the United Kingdom, March 2020. Euro Surveill 2020;25;2000433.
[6] Tostmann A, Bradley J, Bousema T, Yiek WK, Holwerda M, Bleeker-Rovers C, et al. Strong associations and moderate predictive value of early symptoms for SARS-CoV-2 test positivity among healthcare workers, the Netherlands, March 2020. Euro Surveill 2020;25;2000508.

[7] Chatterjee P, Anand T, Singh KJ, Rasaily R, Singh R, Das S, et al. Healthcare workers \& SARS-CoV-2 infection in India: a case-control investigation in the time of COVID-19. Indian J Med Res 2020;151;459-67.

[8] Zhou P, Huang Z, Xiao Y, Huang X, Fan XG. Protecting Chinese healthcare workers while combating the 2019 novel coronavirus. Infect Control Hosp Epidemiol 2020;41;745-6.

[9] Huang L, Zhang X, Zhang X, Wei Z, Zhang L, Xu J, et al. Rapid asymptomatic transmission of COVID-19 during the incubation period demonstrating strong infectivity in a cluster of youngsters aged 16-23 years outside Wuhan and characteristics of young patients with COVID-19: a prospective contact-tracing study. J Infect 2020;80;e1-e13.

[10] Eckerle I, Meyer B. SARS-CoV-2 seroprevalence in COVID-19 hotspots. Lancet 2020;396;514-15.

[11] Dall C. Antibody tests may hold clues to COVID-19 exposure, immunity—but it's complicated. CIDRAP. 2020. Available from: https://www.cidrap.umn.edu/news-perspective/2020/04/ antibody-tests-may-hold-clues-covid-19-exposure-immunityits-complicated (accessed August 12, 2020).

[12] Xu X, Sun J, Nie S, Li H, Kong Y, Liang M, et al. Seroprevalence of immunoglobulin $\mathrm{M}$ and $\mathrm{G}$ antibodies against SARS-CoV-2 in China. Nat Med 2020;26;1193-5.

[13] Korth J, Wilde B, Dolff S, Anastasiou OE, Krawczyk A, Jahn M, et al. SARS-CoV-2-specific antibody detection in healthcare workers in Germany with direct contact to COVID-19 patients. J Clin Virol 2020;128;104437.

[14] Fusco FM, Pisaturo M, Iodice V, Bellopede R, Tambaro O, Parrella G, et al. COVID-19 among healthcare workers in a specialist infectious diseases setting in Naples, Southern Italy: results of a cross-sectional surveillance study. J Hosp Infect 2020;105; $596-600$.

[15] Schlagenhauf P, Grobusch MP, Maier JD, Gautret P. Repurposing antimalarials and other drugs for COVID-19. Travel Med Infect Dis $2020 ; 34 ; 101658$.

[16] Bienvenu AL, Marty AM, Jones MK, Picot S. Systematic review of registered trials of Hydroxychloroquine prophylaxis for COVID-19 health-care workers at the first third of 2020. One Health 2020;10;100141.

[17] National ICMR Taskforce for COVID-19. Advisory on the use of hydroxy-chloroquine as prophylaxis for SARSCoV-2 infection. 2020. Available from: https://www.mohfw. gov.in/pdf/AdvisoryontheuseofHydroxychloroquinasprophy laxisforSARSCoV2infection.pdf (accessed August 12, 2020).

[18] Mitjà O, Corbacho-Monné M, Ubals M, Alemany A, Suñer C, Tebé C, et al. A cluster-randomized trial of hydroxychloroquine for prevention of Covid-19. N Engl J Med 2021;384; 417-27.

[19] Boulware DR, Pullen MF, Bangdiwala AS, Pastick KA, Lofgren SM, Okafor EC, et al. A randomized trial of hydroxychloroquine as postexposure prophylaxis for Covid-19. N Engl J Med 2020;383;517-25

[20] Grau-Pujol B, Camprubí D, Marti-Soler H, Fernández-Pardos M, Guinovart C, Muñoz J. Pre-exposure prophylaxis with hydroxychloroquine for high-risk healthcare workers during the COVID-19 pandemic: a structured summary of a study protocol 
for a multicentre, double-blind randomized controlled trial. Trials 2020;21;688.

[21] Rajasingham R, Bangdiwala AS, Nicol MR, Skipper CP, Pastick KA, Axelrod ML, et al. Hydroxychloroquine as pre-exposure prophylaxis for Coronavirus Disease 2019 (COVID-19) in healthcare workers: a randomized trial. Clin Infect Dis 2020; ciaa1571.

[22] Al-Bari MAA. Targeting endosomal acidification by chloroquine analogs as a promising strategy for the treatment of emerging viral diseases. Pharmacol Res Perspect 2017;5;e00293.

[23] Bhattacharya R, Chowdhury S, Nandi A, Mukherjee R, Kulshrestha M, Ghosh R, et al. Pre-exposure hydroxychloroquine prophylaxis for COVID-19 in healthcare workers: a retrospective cohort. Int J Res Med Sci 2020;9;89-96.

[24] Galvis V, Spinelli FR, Tello A, Sossa CL, Higuera JD, Gómez ED, et al. Hydroxychloroquine as prophylaxis for coronavirus
SARS-CoV-2 infection: review of the ongoing clinical trials. Arch Bronconeumol 2020;56;606-8.

[25] Goenka MK, Afzalpurkar S, Goenka U, Das SS, Mukherjee M, Jajodia S, Shah BB, et al. Seroprevalence of COVID-19 amongst health care workers in a tertiary care hospital of a metropolitan City from India. J Assoc Physicians India 2020;68;14-19.

[26] Khurana A, Kaushal GP, Gupta R, Verma V, Sharma K, Kohli M. Prevalence and clinical correlates of COVID-19 outbreak among health care workers in a tertiary level hospital in Delhi. medRxiv 2020 [Preprint].

[27] Stricker RB, Fesler MC. Flattening the risk: pre-exposure prophylaxis for COVID-19. Infect Drug Resist 2020;13;3689-94.

[28] Banaji M. What do the Delhi and Mumbai sero-survey results tell us about covid-19 in India? The Wire. Available from: https://thewire.in/health/delhi-mumbai-covid-19-coronavirusseroprevalence-survey-results (accessed August 12, 2020). 